\title{
Microbiology Susceptibility Baseline Flag
}

National Cancer Institute

\section{Source}

National Cancer Institute. Microbiology Susceptibility Baseline Flag. NCI Thesaurus. Code C87926.

An indication or description of a baseline value within a microbiology susceptibility assessment. 